\title{
MODELO DE FACTORES QUE AFECTAN EL INTERÉS INDIVIDUAL DE LOS ESTUDIANTES EN LAS CIENCIAS BÁSICAS: DISEÑO Y VALIDACIÓN DE UN CUESTIONARIO

\author{
MODEL OF FACTORS AFFECTING THE INDIVIDUAL INTEREST OF \\ STUDENTS IN BASIC SCIENCES: DESIGN AND VALIDATION OF A
} QUESTIONNAIRE
}

\author{
Raúl Alberto García Castro \\ rgarciac@unjbg.edu.pe \\ Martín Pedro Llapa Medina \\ mllapam@unjbg.edu.pe \\ Willian Bartesaghi Aste \\ wbartesaghia@unjbg.edu.pe
}

Departamento de Ciencias Formales y Naturales.

Universidad Nacional Jorge Basadre Grohmann, Tacna (Perú)

Recibido: $11 / 11 / 2020$

Aceptado: 28/05/2021

\begin{abstract}
Resumen:
El propósito fue diseñar y validar un instrumento para medir a un conjunto de factores endógenos y exógenos que afectan el interés individual de los estudiantes en el aprendizaje de las ciencias básicas. Primeramente, se elaboró un modelo multivariado de factores y luego se diseñó el cuestionario, que fue sometido a validación de contenido con la participación de jueces y una prueba piloto. La validación de constructo se hizo mediante el análisis factorial exploratorio, con una muestra de 274 alumnos de educación secundaria, se identificaron cinco dimensiones latentes. El análisis factorial confirmatorio se realizó con una muestra de 336 alumnos. Los resultados muestran evidencias óptimas del cuestionario que corroboran su validez con 18 ítems. Es un instrumento práctico que permite realizar indagaciones empíricas sobre los efectos de cada uno de los factores que afectan el interés individual mediante las valoraciones perceptivas de los alumnos, dando lugar a diagnósticos multivariados.
\end{abstract}

Palabras clave: factores endógenos y exógenos, interés hacia las ciencias básicas, validación. 


\begin{abstract}
:
The purpose was to design and validate an instrument to measure a set of endogenous and exogenous factors that affect the individual interest of students in learning basic sciences. First, a multivariate factor model was developed and then the questionnaire was designed, which was submitted to content validation with the participation of judges and a pilot test. The construct validation was done through exploratory factor analysis, with a sample of 274 secondary school students, five latent dimensions were identified. The confirmatory factor analysis was carried out with a sample of 336 students. The results show optimal evidence of the questionnaire that corroborates its validity with 18 items. It is a practical instrument that allows empirical inquiries to be made about the effects of each of the factors that affect individual interest through perceptual evaluations of the students, giving rise to multivariate diagnoses.
\end{abstract}

Keywords: endogenous and exogenous factors, interest in basic sciences, validation.

\title{
1. Introducción
}

El estudio de la Física, Química, Biología y las Matemáticas en la educación secundaria posibilita revisar los principios fundamentales de la naturaleza, entender el sentido de los fenómenos naturales o de la realidad misma, sentando las bases de una sociedad científicamente alfabetizada con poder crítico sobre las cuestiones científicas. Su aprendizaje se basa principalmente en procesos que requieren la aplicación de fundamentos matemáticos, hecho que desanima a los estudiantes, porque les resulta complicado y terminan por rechazarlas (Solbes et al., 2007). En muchos países, principalmente en sur américa, la educación científica ha sido declarada una meta prioritaria. Recientemente se han desarrollado procesos de reforma de los sistemas de formación docente, incluyendo iniciativas de reconversión y acreditación de las instituciones formadoras. En la educación secundaria, se vienen actualizando los currículos (Furman, 2018). No obstante, las evaluaciones nacionales e internacionales muestran resultados negativos en el aprendizaje de las ciencias (Solves y Furió, 207; Busquets, et al., 2016).

Existe la sensación de que los esfuerzos para mejorar el aprendizaje caminan lentos o simplemente no dan resultados. En este problema el interés individual del estudiante hacia las ciencias básicas es un factor de gran valor y juega un papel importante (Whitelegg y Parry, 1999). Por qué, el aprendizaje tiene una connotación individual, nace como el resultado del esfuerzo propiciado por el propio estudiante. Este aprende si siente interés en un determinado conocimiento, movilizando su actividad mental hacia ese conocimiento $u$ objeto y lo mantiene relativamente en el tiempo (Schiefele, 1991; 1992). El interés tiene el poder de despertar el deseo de implicarse en las actividades de aprendizaje (Boekaerts y Boscoso, 2002), se considera que es una fuente de la motivación intrínseca (Hidi y Renninger, 2006).

El interés individual es un concepto multidimensional frecuentemente confundido con los constructos de motivación y actitud. Con relación a la motivación, el interés es uno de sus componentes principales, y es considerado el combustible que mueve a la misma (Schiefele, 1991; 1992). La actitud es una respuesta subjetiva del individuo que puede ser positiva o negativa frente a los estímulos de su entorno, es una sensación de agrado o disgusto que siente el estudiante hacia el aprendizaje de las ciencias (Mohd, et al., 2018). El interés es un factor influyente en la actitud. Como se puede apreciar, tanto la motivación como la actitud son conceptos con diferente significado y no deben ser confundidos con el interés personal (Krapp y Prenzel, 2011).

Por su naturaleza, el interés individual es considerado una disposición psicológica de la persona a preferir una actividad o acción, con relativa estabilidad (Krapp et al., 1992; Hidi, 2006), 
influenciada por múltiples factores. Es una variable que depende de distintos factores que explican la disposición hacia el aprendizaje del estudiante. Las variables que influyen sobre el interés individual, también se denominan factores, que se agrupan en factores endógenos y factores exógenos, pueden ser de orden social, cognitivo y emocional (Moreira, 2009; Marba y Márquez, 2012). En general, un factor es un elemento que causa un efecto sobre otro factor (Pérez y Gardey, 2009) independientemente del lugar, espacio o condición social.

En nuestra investigación, los factores exógenos, también denominados variables extrínsecas, son aquellos que no dependen del alumno, no están bajo su control y provienen de su entorno (Romero y Hernández, 2019). Por ejemplo: los métodos de enseñanza, la retroalimentación, los materiales didácticos, el laboratorio, etc. Los factores endógenos o variables intrínsecas son aquellas que se movilizan al interior de la estructura cognitiva y afectiva del estudiante, es decir, nacen en su interior o se originan en sus raíces internas (Romero y Hernández, 2019). Por ejemplo: la memoria, la atención, los hábitos de estudio, etc.

En la literatura científica, encontramos diferentes estudios, Cury et al. (1969) evaluaron la influencia de factores individuales y contextuales en el interés de las adolescentes en la educación física escolar, utilizando la escala de clima motivacional, sub-escalas de competencias y un cuestionario de percepción del éxito. Trumper (2006) investigó sobre los factores que afectan el interés personal de los estudiantes israelís en la biología, y utilizó el cuestionario ROSE (Schreiner y Sjoberg, 2004) con escala tipo Likert, con cuatro niveles de respuesta y 250 ítem. Identificó tres factores sobre el interés individual: la imagen negativa de las ciencias, comportamiento de los profesores y los planes de estudios. Solbes et al. (2007), aplicaron un cuestionario y entrevistas a alumnos de educación secundaria, comprobando la influencia de cuatro factores que afectan el interés de los alumnos: la imagen la valoración negativa de la ciencia, el tema del género y la enseñanza de las ciencias.

Otro trabajo relacionado con el análisis de factores sobre el interés individual fue realizado por Ito y McPherson (2018) quienes elaboraron un cuestionario con cuatro factores sociales: pertenencia social, capacidad de pertenencia, pertenencia a la escuela y autoeficacia. Como resultados del estudio determinaron que existen diferencias significativas en la influencia que ejercen estas variables, entre varones y mujeres, aunque, concluyen que ninguna variable fue significativa en el interés. Derek (2018) entrevistó a profesores y 60 alumnos para identificar factores asociados al interés individual, identifico cuatro factores: influencias situacionales en las lecciones, auto-concepto científico, interés individual en las lecciones de las ciencias e interés individual en las ciencias.

En las investigaciones publicadas abordan la temática analizando uno o más de dos factores endógenos o exógenos, relacionándolos con el interés. En otros trabajos, la selección de los factores que afectan el interés individual del estudiante parten del análisis bibliográfico que selecciona el investigador. No se encontraron instrumentos que permitan un análisis con mayor cobertura de factores relacionándolos con este constructo. Por tanto, cabe la posibilidad de contar con un instrumento que sirva como una herramienta de análisis multivariable, con capacidad de medir una amplia gama de variables que afectan el interés individual de los alumnos.

La presente investigación tiene como objetivo, desarrollar un instrumento actualizado con propiedades psicométricas, para medir e identificar los factores endógenos y exógenos que afectan el interés individual de los estudiantes hacia el aprendizaje de las ciencias básicas. La percepción de los estudiantes, así, como la opinión de los profesores y expertos, son fuentes importantes, para identificar estos factores. Con este objetivo, mediante la indagación exploratoria se busca construir un modelo teórico de factores endógenos y exógenos, para luego, proceder a su validación de constructo. 


\section{Método}

\subsection{Procedimiento}

La investigación se desarrolló en dos etapas: la primera tiene como objetivo identificar un modelo o conjunto de factores endógenos y exógenos que afectan el interés individual de los alumnos. En la segunda etapa se diseñó y valido el cuestionario multivariado, tomando como muestra a los alumnos. Quienes expresaron sus valoraciones subjetivas sobre las variables expuestas en el cuestionario de factores. Esto permitió obtener un instrumento dimensionado que contiene un conjunto de factores endógenos y exógenos, para ser utilizados en el contexto de la educación secundaria.

En la primera etapa se aplicó la técnica de focus group, realizando entrevistas en profundidad a los alumnos y profesores, con matices sobre las causas y valoraciones del fenómeno (Barbour, 2013). Esta técnica también fue aplicada en otros estudios similares (Romero y Hernández 2019; Tarabini, Curran et al., 2015). Los grupos se conformaron con 10 alumnos y de 4-5 los grupos de los profesores. Las entrevistas se realizaron con un protocolo de desarrollo pre establecido, estando a cargo de un grupo de pedagogos y psicólogos, quienes realizaron preguntas motivadoras a fin de registrar la opinión de los participantes. Cada entrevista duró entre 50 a 60 minutos. El registro de la información se realizó mediante una ficha de entrevista abierta, tanto, para los profesores como para los alumnos, y las sesiones fueron grabadas y se registraron en vídeos. También se realizaron entrevistas personales a los especialistas nacionales y extranjeros, para lo cual, se propiciaron reuniones de trabajo visitando las localidades correspondientes. Estas entrevistas duraron entre 2 y 3 horas, en días alternos.

El procesamiento de datos en la primera etapa (cualitativa) se realizó siguiendo los pasos del método de la Teoría Fundada (Strauss y Corbin, 1990). Consistió en tomar los datos provenientes de los focus group y las entrevistas a los especialistas, con los que se procedió a la codificación abierta, reuniendo un conjunto de frases típicas como respuestas de los entrevistados. Las frases son literales (San Martín, 2014), la similitud que ofrecían las respuestas permitió obtener una lista de códigos (frases), que se interpretaron y categorizaron, logrando organizar un resumen de ideas (Flick, 2007). Seguidamente se realizó la codificación axial, identificándose frases literales recurrentes que dan origen a los códigos comunes. Finalmente se realizó el proceso de codificación selectiva, que consistió en la condensación de todos los productos para obtener términos selectivos que representan una estructura conceptual que integra la realidad expresada por los sujetos investigados (Strauss y Corbin, 1990). Este proceso, unido al criterio de la mayor frecuencia de los códigos en los sujetos observados, permitió identificar los códigos finales que, como unidades seleccionadas, convergen en la unidad central de la investigación que se denominan: factores endógenos y exógenos.

En la segunda etapa, el cuestionario se sometió a las pruebas estadísticas del análisis factorial exploratorio (AFE), con el propósito identificar una estructura de factores subyacentes a partir de un conjunto de ítems (Blalock, 1966; Martínez, 1995). El análisis factorial confirmatorio (AFE) se estimó sobre la matriz de correlaciones policóricas, para variables categóricas, con el programa FACTOR versión 10.8 (Lorenzo-Seva y Ferrando, 2006). Se utilizó el método de extracción Minimum Rank Factor Analysis (Ten Berge y Kiers, 1991), con rotación oblicua que permite identificar el número de ítems correlacionados por factor, como es el caso de la rotación Promax (Dien, 2010; Hendrickson y White, 1964; Tabachnick y Fidell, 2007).

Para verificar que el ítem pertenece a una dimensión se consideró una carga factorial por encima de 0.3 (Hogarty et al., 2005). Así mismo, se verificó que el número de ítems por factor fuese mayor a tres (ideal 3 o 4 variables por factor), aunque en la práctica incluso dos variables podrían ser suficientes (Velicer y Fava, 1998). Para verificar la correlación satisfactoria entre los ítems (magnitudes que fluctúan entre 0 y1) se aplicó el coeficiente (KMO) de Kaiser-Mayer-Olikin 
(Kaiser, 1970; Thompson, 2008). La prueba de esfericidad de Bartlett permite verificar si la matriz de datos es apropiada para un análisis factorial exploratorio (Bartlett, 1950, 1951; Gorsuch, 1973; Yanai y Ichikawa, 2007). La evaluación de la bondad de ajuste del modelo factorial se realizó mediante el cuadrado medio de la raíz de residuos (RMSR) y el valor medio esperado de RMSR para un modelo aceptable (Kelley, 1935; Harman, 1962) y el porcentaje acumulado de la varianza (Timmerman y Lorenzo-Seva, 2011).

A los 20 días se aplicó el cuestionario nuevamente y con esta información se realizó el análisis factorial confirmatorio (AFC). Este procedimiento posibilitó una mayor contrastación de las hipótesis especificadas (Bollen, 1989). El análisis del ajuste del modelo se realizó utilizando el software para análisis de datos categóricos Mplus (Muthén, 2004). Los estimadores aplicados se basaron en correlaciones tetracóricas para variables categóricas (Muthén,1998; Muthen, 2004). Seguidamente con el programa $\mathrm{R}$ estudio se realizó el análisis de fiabilidad para verificar su consistencia interna de cada uno de los factores.

\subsection{Participantes}

Para la primera etapa se tomó una muestra estudiantes y docentes de ciencias de 22 instituciones educativas públicas y privadas de la Región de Tacna (Perú). Se seleccionaron al azar un total de 220 estudiantes, a razón de 10 estudiantes por cada institución del al 10 al 50 año de secundaria. La muestra está compuesta por alumnos de ambos sexos y cuyas edades fluctúan entre 11 y 17 años, con un promedio de 14.9 años y SD $=0.95$. En el caso de los profesores, se seleccionaron 99 en total, 35 de matemáticas y 64 de Ciencia, Tecnología y Ambiente (Química, Física y Biología). También se contó con 8 especialistas del sector educación de la Región de Tacna y 6 de cuatro regiones del Perú, quienes se desempeñan como supervisores del área de Ciencia, Tecnología y ambiente. Así mismo, participaron 8 especialistas internacionales de España y Argentina, en cuyo caso se seleccionaron a profesionales que ejercen la docencia Universitaria en educación y realizan trabajos de investigación, relacionados a las ciencias básicas.

Para la segunda etapa se seleccionaron aleatoriamente dos muestras de alumnos, pertenecientes a 22 instituciones educativas de la Región de Tacna (Perú). Se conformaron grupos de entre 12 y 17 alumnos por institución. Con la primera muestra se realizó el análisis factorial exploratorio, compuesta por 274 alumnos del $1^{\circ}$ al 5 o año, donde el $52.2 \%$ fueron mujeres y el $47.8 \%$ varones, y cuyas edades fluctúan entre 12 y 18 años, con un promedio de edad de 15,1 años y SD = 1.038. La segunda muestra se utilizó para el AFC, se seleccionó al cabo de 20 días, y está conformada por 336 alumnos, donde el 53.5\% fueron mujeres y el $46.5 \%$ varones; las edades fluctúan entre 12 y 19 años, con un promedio de 15,4 años. También se contó con seis especialistas para la revisión del cuestionario y una muestra piloto de 50 alumnos.

\section{Resultados}

En este apartado se presentan los resultados de la primera y segunda etapa de la investigación. En la primera se presenta el modelo de factores endógenos y exógenos. En la segunda etapa se valida el cuestionario mediante el AFE, el AFC y la prueba de fiabilidad.

\subsection{Identificación del modelo de factores endógenos y exógenos (etapa cualitativa)}

Como resultado de la codificación abierta y axial, se identificaron entre los alumnos 509 frases literales que dieron origen a 36 códigos. Con los profesores se identificaron 221 frases que dieron origen a 29 códigos. Entre los especialistas de las Regiones del Perú y el extranjero, se registraron 96 frases que dieron origen a 16 códigos. La codificación selectiva, bajo la selección del criterio de la mayor frecuencia permitió seleccionar 21 códigos. Conforme a la naturaleza conceptual de cada uno de ellos se identificaron 12 factores exógenos y 9 factores endógenos, 
los mismos que, sobre la base de la teoría existente y las manifestaciones conductuales de los sujetos observados, han sido conceptualizados dando lugar al modelo de factores endógenos y exógenos. Los resultados finales se presentan en la tabla siguiente:

Tabla 1

Modelo de factores endógenos y exógenos

\begin{tabular}{|c|c|c|c|}
\hline $\mathrm{N}^{\circ}$ & Patrón & Factor & Concepto \\
\hline 1 & & $\begin{array}{l}\text { Métodos y técnicas } \\
\text { de enseñanza }\end{array}$ & $\begin{array}{l}\text { Organización racional de procedimientos para la } \\
\text { enseñanza. Comprende uso del tiempo, } \\
\text { materiales, equipos, y las circunstancias. }\end{array}$ \\
\hline 2 & & Apoyo familiar & $\begin{array}{l}\text { Ayuda que brindan los padres de familia a sus } \\
\text { hijos para el aprendizaje, ya sea emocional o } \\
\text { colaborando para encontrar las soluciones ante } \\
\text { un problema académico. }\end{array}$ \\
\hline 3 & & $\begin{array}{l}\text { Problemas } \\
\text { familiares }\end{array}$ & $\begin{array}{l}\text { Entorno familiar inestable y a veces conflictivo, } \\
\text { ocasionado por la ruptura de las relaciones entre } \\
\text { el padre y la madre, debido a problemas de } \\
\text { violencia, desempleo, alcoholismo, etc. }\end{array}$ \\
\hline 4 & & Bulla escolar & $\begin{array}{l}\text { Ruido proveniente del alboroto o griterío } \\
\text { producido por los alumnos en un salón de clases } \\
\text { (Castro y Morales, 2015). }\end{array}$ \\
\hline 5 & & Equipamiento & $\begin{array}{l}\text { Disponibilidad en laboratorios o salas de } \\
\text { aprendizaje de instrumentos, materiales y } \\
\text { equipos, para la realización de las prácticas } \\
\text { experimentales (Insauti, y Merino, 200). }\end{array}$ \\
\hline 6 & $\begin{array}{l}\text { Factores } \\
\text { exógenos }\end{array}$ & Matematización & $\begin{array}{l}\text { Uso predominante de estrategias de resolución de } \\
\text { problemas con ejercicios matemáticos en la } \\
\text { enseñanza de las ciencias básicas, dificultando su } \\
\text { comprensión y vínculo con la realidad práctica } \\
\text { (Busquets, et al., 2016) }\end{array}$ \\
\hline 7 & & Tiempo de estudio & $\begin{array}{l}\text { Número de horas por semana programadas por } \\
\text { cada asignatura, conforme el plan de estudios. }\end{array}$ \\
\hline 8 & & Chateo & $\begin{array}{l}\text { Es el espacio de comunicación sostenido vía } \\
\text { dispositivos móviles o a través de un ordenador. }\end{array}$ \\
\hline 9 & & $\begin{array}{l}\text { Experimentación } \\
\text { en laboratorio }\end{array}$ & $\begin{array}{l}\text { Tiempo en número de horas dedicados a las } \\
\text { actividades didácticas en el laboratorio. }\end{array}$ \\
\hline 10 & & Valoración familiar & $\begin{array}{l}\text { Grado de importancia que le otorgan los padres } \\
\text { de familia al estudio de las ciencias(Bazán et al., } \\
\text { 2013). }\end{array}$ \\
\hline 11 & & Material didáctico & $\begin{array}{l}\text { Conjunto de recursos de apoyo que utiliza el } \\
\text { profesor para el proceso de aprendizaje, incluyen } \\
\text { materiales bibliográficos, separatas, vídeos, etc. }\end{array}$ \\
\hline 12 & & Grupos de estudio & $\begin{array}{l}\text { Conjunto de alumnos que se auto convocan y } \\
\text { establecen sus reglas de trabajo para repasar las } \\
\text { lecciones aprendidas en clases (Ruiz et al., 2012). }\end{array}$ \\
\hline
\end{tabular}


Factores

17
Atención selectiva

Predisposición

Hábitos de estudio

\section{Auto-concepto académico}

Técnicas de estudio

\section{(}

Conjunto de procedimientos concretos que

Conjunto de palabras y frases especializadas, que Lenguaje científico conforman un vocabulario específico en cada una de las materias de las ciencias básicas.

Conjunto de conocimientos que posee el alumno,

Conocimientos previos

Percepción de utilidad intelectuales para aprender las ciencias básicas (Papalia et al., 2006). almacenados en su memoria, producto de sus experiencias previas de aprendizaje (Ausubel, 1973).

Interpretación que realiza el alumno sobre el beneficio que le aporta en la actualidad y en el futuro el aprendizaje de las ciencias básicas
Habilidad para focalizar sus sentidos sensoriales ante los estímulos provenientes del proceso de aprendizaje, inhibiendo a los estímulos distractores.

Voluntad para aprender, debido a su actitud mental favorable hacia las ciencias básicas.

Comportamiento repetitivo en la acción de estudiar, ya sea repasando las lecciones, investigando o haciendo las tareas (Cartagena, 2008).

Capacidad de retener y recuperar la información, proveniente de experiencias o eventos relacionados con el proceso de aprendizaje (Etchepareborda, 2005).

Valoración de sí mismo, sobre las capacidades (Claves, 2005; Jenkins y Pell, 2006).

\subsection{Diseño del cuestionario}

La construcción del cuestionario se elaboró sobre la base de la matriz condicionada que agrupa los 21 factores seleccionados (tabla 1). Para cada factor se redactó un ítem tomando en cuenta las reglas de diseño para cuestionarios de tipo Likert (De Vellis, 2012; Furr, 2011). La escala seleccionada es de 1 a 5 donde 1 representa "nada", 2 "un poco", 3 "regular", 4 "bastante" y 5 "mucho" (Hernández et al., 2000). Las respuestas del cuestionario se realizan mediante la valoración personal del alumno motivadas por la pregunta: ¿Cuánto afecta cada uno de los factores a tu interés personal en el aprendizaje de las ciencias? El cuestionario se sometió a la opinión de expertos para evaluar la concordancia entre los enunciados y los factores seleccionados. Sus apreciaciones contribuyeron a precisar los factores y organizar los conceptos, además observaron la redacción de los ítems, los cuales, fueron corregidos. Seguidamente se aplicó una prueba piloto con 50 alumnos de educación secundaria del 3ㅇal 5 grado de estudios, solicitándoles que evaluasen la comprensión de los enunciados. Producto de este proceso se corrigió la redacción de 6 ítems, concluyendo la etapa preliminar. 


\subsection{Resultados del análisis factorial exploratorio (AFE)}

Previo al análisis factorial exploratorio (AFE) se verifico la correlación entre los ítems, la prueba proporcionó un valor de Kaiser-Meyer- Olkin (KMO) de 0.807, que se interpreta como un nivel alto (Beavers et al., 2013). La prueba de esfericidad de Bartlett's $=1369.4$ ( $d f=210 ; p=0.00001$ ), representa un nivel significativo. Ambos resultados indican que es ade-cuado hacer un AFE. Mediante el método de extracción Minimum Rank Factor Analysis (Ten Berge y Kiers, 1991), con rotación oblicua, se identificó una solución de 6 factores emergentes. Adicionalmente se realizaron pruebas de descarte con 4, 5 y 7 factores, las soluciones no satisfacían el modelo teórico de factores endógenos y exógenos. En consecuencia, se descartaron estas soluciones quedándonos con la solución sugerida por el programa de 6 factores subyacentes (dimensiones). La solución explica un $\mathbf{7 8 . 8 7 \%}$ de la varianza total, cuyos factores se agruparon conforme se muestra en la tabla 2.

Tabla 2

Coeficientes del Análisis Exploratorio

\begin{tabular}{|c|c|c|c|c|c|c|}
\hline Ítems & F1 & F2 & F3 & F4 & F5 & F6 \\
\hline ES1 & 0.621 & & & & & \\
\hline ES2 & 0.432 & & & & & \\
\hline ES3 & 0.408 & & & & & \\
\hline ES4 & 0.747 & & & & & \\
\hline CF5 & & 0.551 & & & & \\
\hline CF6 & & 0.982 & & & & \\
\hline CF7 & & 0.486 & & & & \\
\hline CF8 & & 0.597 & & & & \\
\hline CE9 & & & 0.474 & & & \\
\hline CE10 & & & 0.658 & & & \\
\hline CE11 & & & 0.694 & & & \\
\hline CE12 & & & 0.777 & & & \\
\hline CE13 & & & 0.804 & & & \\
\hline
\end{tabular}

EM14

En la tabla 2 se puede apreciar que los ítems son $>0.3$, bajo este criterio no se eliminan ítems. A pesar de ello, se observa que la dimensión 4 está compuesto por dos ítems teóricamente distintos y pueden resultar controversiales al momento de juzgar esta dimensión, por lo que 
resulta conveniente eliminar estos ítems. Así mismo, se juzga conveniente eliminar el ítem 17, de la dimensión 5, debido a que teóricamente tiene un significado distinto a los ítems 16 y 18.

Cada una de las dimensiones subyacentes identificados toman una denominación conforme a la naturaleza de los ítems que lo agrupan, los resultados se pueden apreciar en la tabla 4.

\subsection{Análisis factorial confirmatorio (AFC)}

Con el objeto de corregir o corroborar las agrupaciones de los ítems del cuestionario se aplicó el AFC, que posibilita una mayor contrastación de las hipótesis especificadas (Bollen, 1989). EI análisis del ajuste del modelo (Tabla 2) se realizó con la ayuda del programa Mplus (Muthén, 2004). Los estimadores aplicados se basaron en correlaciones tetracóricas para variables categóricas (Muthén, 2004).

Tabla 3

Resultados estadísticos de ajuste robustos para los modelos de AFC

\begin{tabular}{cccccccc}
\hline Modelo & $\chi 2$ & g.l & p-valor & CFI & TLI & RMSE & SRMR \\
\hline 1 & 367.749 & 174 & 0.000 & 0.90 & 0.92 & 0.063 & 0.069 \\
\hline
\end{tabular}

Los resultados de la tabla 3, confirman que el modelo presenta un ajuste aceptable a los datos: el índice de ajuste comparativo $\mathrm{CFI}=0.90$ está ligeramente por debajo del nivel deseado y el TLI $=0.92$, por encima de 0.9. El error cuadrado medio de aproximación RMSEA $=0.063$, se considera un modelo plausible (Kaplan, 2009; Mulaik, 2009). Los resultados de las cargas factoriales de los ítems se presentan en la tabla 4.

Tabla 4

Cargas factoriales de los ítems (AFC)

\begin{tabular}{|c|c|c|c|}
\hline Dimensión & Ítems & $\begin{array}{l}\text { Carga } \\
\text { Factorial }\end{array}$ & Enunciado del ítems \\
\hline \multirow{4}{*}{$\begin{array}{l}\text { Dimensión } 1 \\
\text { Entorno social }\end{array}$} & ES1 & 0.416 & Los problemas familiares. \\
\hline & ES2 & 0.484 & La bulla que hacen los compañeros en clases. \\
\hline & ES3 & 0.54 & El interés de mis padres por las ciencias. \\
\hline & ES4 & 0.481 & $\begin{array}{l}\text { Disposición de los compañeros para repasar en } \\
\text { grupo. }\end{array}$ \\
\hline \multirow{4}{*}{$\begin{array}{l}\text { Dimensión 2: } \\
\text { Capacidad de } \\
\text { focalización }\end{array}$} & CF5 & 0.462 & $\begin{array}{l}\text { La atención y concentración, cuando se trata de } \\
\text { estudiar. }\end{array}$ \\
\hline & CF6 & 0.433 & $\begin{array}{l}\text { Mi predisposición para aprender las matemáticas y } \\
\text { CTA. }\end{array}$ \\
\hline & CF7 & 0.487 & Los hábitos para repasar las lecciones. \\
\hline & CF8 & 0.447 & $\begin{array}{l}\text { Sentimientos de imposibilidad para aprender las } \\
\text { matemáticas y CTA. }\end{array}$ \\
\hline \multirow{3}{*}{$\begin{array}{l}\text { Dimensión 3: } \\
\text { Competencias } \\
\text { para el estudio }\end{array}$} & CE9 & 0.487 & Mi capacidad de memoria \\
\hline & CE10 & 0.679 & Mis técnicas de aprendizaje. \\
\hline & CE11 & 0.580 & $\begin{array}{l}\text { Los términos técnicos que usan para las } \\
\text { matemáticas y CTA. }\end{array}$ \\
\hline
\end{tabular}




\begin{tabular}{|c|c|c|c|}
\hline & CE12 & 0.664 & $\begin{array}{l}\text { Mis conocimientos previos de las matemáticas y } \\
\text { CTA. }\end{array}$ \\
\hline & CE13 & 0.598 & $\begin{array}{l}\text { Utilidad de las ciencias matemáticas y CTA, en el } \\
\text { futuro. }\end{array}$ \\
\hline \multirow{2}{*}{$\begin{array}{l}\text { Dimensión 4: } \\
\text { Estrategias } \\
\text { instruccionales }\end{array}$} & EIN14 & 0.707 & Los métodos y técnicas del docente. \\
\hline & EIN15 & 0.557 & El Material didáctico que usa el profesor. \\
\hline \multirow{3}{*}{$\begin{array}{l}\text { Dimensión 5: } \\
\text { Tiempo de } \\
\text { estudio }\end{array}$} & TE16 & 0.35 & Pocas horas de clases, la clase es muy rápida. \\
\hline & TE17 & 0.44 & $\begin{array}{l}\text { Uso de las redes sociales (Facebok, wahatsApp, } \\
\text { otros) con fines de distracción. }\end{array}$ \\
\hline & TE18 & 0.403 & Las prácticas en laboratorio. \\
\hline
\end{tabular}

Los índices de estimación (Tabla 4) indican que los factores del modelo tienen cargas mayores > 0.3 , por lo que, se juzga correcto mantener en el modelo todos los ítems. Sin embargo, dejamos constancia que el ítem TE18 contiene una carga factorial baja, por lo que podría ser eliminado a fin de favorecer la optimización del modelo.

Tabla 5

Matriz de correlación entre componentes (dimensiones)

\begin{tabular}{llllllllll}
\hline D2 & D3 & D3 & D4 & D4 & D4 & D5 & D5 & D5 & D5 \\
D1 & D1 & D2 & D1 & D2 & D3 & D1 & D2 & D3 & D4 \\
\hline 0.46 & 0.62 & 0.60 & 0.46 & 0.401 & 0.39 & 0.602 & 0.60 & 0.65 & 0.54
\end{tabular}

En la tabla 5 se puede observar que los componentes del modelo se encuentran correlacionados, con cargas factoriales entre dimensiones observadas $>0.3$. La correlación con la más baja carga factorial (0.39) es la compuesta por competencias para el estudio (D4) y estrategias instruccionales (D3). La correlación más alta (0.65) se muestra entre las competencias para el estudio (D3) y las estrategias institucionales (D5).

\subsection{Resultados del análisis descriptivo y fiabilidad}

El análisis de fiabilidad se realizó con $\mathrm{R}$ Studio para datos ordinales. Los resultados se presentan en la tabla 6 . Se puede observar que las dimensiones alcanzaron coeficientes altos que fluctúan entre 0.79 (Entornos de aprendizaje) y 0.91 (Competencias para el estudio). Todas las dimensiones superan la magnitud de 0.75 , en consecuencia, no se recomienda eliminar elementos. Del análisis de alfa ordinal se puede concluir que el cuestionario y sus dimensiones emergentes se pueden utilizar para realizar estudios sobre los factores endógenos y exógenos en estudiantes de educación secundaria. Los resultados de los intervalos de confianza al $95 \%$ indican que el nivel de fiabilidad en la muestra total es de 0.92 . 
Tabla 6

Resultados descriptivos y fiabilidad

\begin{tabular}{llccc}
\hline $\mathrm{N}^{\circ}$ & Dimensión & $\mathrm{N}^{\circ}$ de ítems & Media & Alfa ordinal \\
\hline 1 & Entorno social de aprendizaje & 4 & 2.69 & 0.79 \\
2 & Capacidades de focalización & 4 & 2.74 & 0.85 \\
3 & Competencias para el estudio & 5 & 3.20 & 0.91 \\
4 & Estrategias instruccionales & 3 & 3.10 & 0.80 \\
5 & Tiempo de estudio escolar & 3 & 2.72 & 0.83 \\
& & Alfa ordinal con los 18 ítems del cuestionario & 0.92 \\
\cline { 2 - 4 }
\end{tabular}

\section{Discusión}

La investigación permitió construir un modelo teórico de factores endógenos y exógenos mediante la investigación a nivel exploratorio. Cada uno de los factores identificados son variables que afectan al interés en el aprendizaje de las ciencias y representan estructuras conceptuales, que se convierten en un aporte importante para la construcción del cuestionario. Su identificación parte de las indagaciones realizadas con los propios alumnos, quienes son actores directos en el proceso de aprendizaje, los docentes de aula y los expertos. Es un instrumento multidimensional que cubre un amplio espectro de factores endógenos y exógenos, con 5 dimensiones latentes, que han sido conceptualizadas.

Los resultados del AFE proporcionan una solución que explica un $78.87 \%$ de la varianza total. Cada dimensión agrupa un conjunto de ítems que dan origen a una estructura conceptual que fueron confirmados mediante el análisis factorial confirmatorio (AFC) y la pruebas confiabilidad. Los resultados corroboran que el cuestionario cumple con los requerimientos psicométricos básicos que propone la teoría para este tipo de instrumentos (Furr y Bacharach, 2008).

La validación del cuestionario permite contar con un instrumento para ser aplicado en alumnos de 11 a 18 años, con el fin de evaluar sus percepciones respecto al impacto que tienen cada uno de los factores endógenos y exógenos en el interés en el aprendizaje de las ciencias básicas. El diseño del cuestionario, valorado con escala tipo Lickert, ofrece una alternativa práctica de fácil aplicación en el contexto educativo. Representa un aporte para los investigadores, gestores educativos, y otros interesados que requieren obtener una visión rápida de los problemas que afectan el aprendizaje de las ciencias básicas.

El estudio de los factores que afectan el interés individual, también ha sido realizado por Derek (2018), quien concluye que el auto-concepto científico, es una variable de importancia y fue reportada en otros contextos: Marsh et al., (2005) en Alemania; Viljaranta et al., (2014) en Finlandia y Cury et al., (1996) en Francia. El investigador también reporta la influencia situacional y el interés individual en las lecciones, como factores que afecta el interés, ambos factores cobren un amplio espectro conceptual, que incluyen una medición sobre enunciados genéricos, como: la participación en equipos de estudio, la atención, las emociones durante el aprendizaje, entre otros. Es una de las pocas investigaciones, donde se identifican los factores relacionados con el interés individual, a través de la indagación con estudiantes y profesores. Sin embargo, a diferencia de la presente investigación, es el autor quien realiza la agrupación de los ítems y luego los somete a una validación de constructo mediante un AFC. Ito y McPeherson (2018) realizaron un estudio de factores que influyen en interés individual de las ciencias, el 
cuestionario incluye el factor de pertenencia social, pertenencia a la escuela, habilidad perteneciente y auto eficacia. Únicamente el factor de autoeficacia guarda similitud con el factor auto-concepto académico con el presente cuestionario.

Hasni (2015) válido un cuestionario donde incluye a los métodos de enseñanza y la auto eficacia. Estos factores son parte del cuestionario validado en la presente investigación, lo cual, corrobora la importancia de estos factores. Otro factor que incluye su cuestionario, es el contexto familiar, el mismo, que está referido a la transmisión cultural de las ciencias que realiza la familia, mediante el uso de programas de televisión, visitas al museo, conversaciones sobre las ciencias, etc. En el cuestionario de factores endógenos y exógenos se mide esta variable, en función de la ayuda práctica que ofrece la familia en la solución de los problemas y necesidades de aprendizaje, lo cual, estable una diferencia significativa en su constructo. Por su parte, Polino (2012) realizo una investigación para explorar los factores que condicionan el interés por las ciencias, en adolescentes. Sus hallazgos dan cuenta de la importancia del uso de recursos pedagógicos, que en el presente cuestionario se denominan métodos de enseñanza, sin embargo, incluye el factor de contenidos curriculares, el mismo, que no es parte del presente cuestionario validado.

Las contribuciones de estas investigaciones señalan una ruta importante en el estudio de los factores que afectan el interés personal. Sus resultados contienen algunos de los 18 factores que componen el presente cuestionario validado. A diferencia del número de factores que ofrecen los instrumentos analizados anteriormente. En este sentido, el cuestionario de factores endógenos y exógenos, es una herramienta práctica que permite a los alumnos de un contexto determinado, identificar entre múltiples factores, aquellos que con mayor fuerza impactan sobre su interés personal. Se basa en la percepción de los propios alumnos, quienes en su condición de usuarios del servicio educativo proporcionan valoraciones subjetivas sobre un conjunto de factores recurrentes. Las mediciones psicométricas que proporciona el cuestionario son sólidas, considerando los resultados de validez de constructo, confiabilidad y estabilidad.

El cuestionario reúne las cualidades de flexibilidad para adaptarlo al nivel de educación primaria o superior, lo que requeriría su adecuación, principalmente en la redacción de los ítems. También, el modelo puede seguir alimentándose con otros factores no previstos que surjan de las propias características socio culturales del contexto en que se requiera aplicar. Por otro lado, los resultados que se obtienen con la aplicación del cuestionario pueden propiciar posibles propuestas o alternativas para enfrentar el problema del desinterés de los alumnos por las ciencias básicas. Finalmente, el cuestionario ofrece una alternativa para utilizarlo como herramienta contextualizada a poblaciones afines a la muestra

Una de las limitaciones del estudio es que el cuestionario ha sido validado con una muestra de alumnos de educación secundaria de la República del Perú, por tanto, es necesario diversificar la naturaleza de la muestra a fin de continuar con su corroboración. También puede discutirse la eliminación o inclusión de alguno de los ítems, ya que el trabajo se entiende como una primera aproximación a la medición de los factores que afectan el interés individual.

\section{Referencias bibliográficas}

Ausubel, D. P. (1973). Algunos aspectos psicológicos de la estructura del conocimiento. Investigaciones sobre el proceso de aprendizaje y la naturaleza de las disciplinas que integran el currículum. El Ateneo.

Barbour, R. (2013). Los grupos de discusión en investigación cualitativa. Ediciones Morata.

Bartlett, M. S. (1950). Tests of significance in factor analysis. British Journal of Psychology, (3), 77-85. 
Bartlett, M. S. (1951). A further note on tests of significance. British Journal of Psychology, (4), $1-2$.

Bazán, A., Backhoff E. y Turullols R. (2016). Participación escolar, apoyo familiar y desempeño en Matemáticas: El caso de México en PISA (2012). Relieve, 22(1), 1-18. https://www.uv.es/RELIEVE/v22n1/RELIEVEv22n1 M5.pdf

Beavers, A. S., Lounsbury,, J. W., Richards J. K., Huck, S. W., Skolits, G. J., y Esquivel, S. L. (2013). Practical Considerations for Using Exploratory Factor Analysis in Educational Research. Practical Assessment, Research \& Evaluation, 18(6), 1-13. http://pareonline.net/getvn.asp?v=18\&n=6

Blalock, H. M. (1966). Estadística Social. Fondo de Cultura Económica.

Boekaerts, M., y Boscolo, P. (2002). Interest and learning, learning to be interested. Learning and Instruction, (12), 375-382.

Bollen, KA. (1989). Structural equations with latent variables. John Wiley \& Sons.

Busquets, T., Silva, M. y Larrosa, P., (2016). Reflexiones sobre el aprendizaje de las ciencias naturales. Nuevas aproximaciones y desafíos. Estudios pedagógicos, 42, (especial), 117135. http://dx.doi.org/10.4067/S0718-07052016000300010

Cartagena, M. (2008). Relación entre la autoeficacia, el rendimiento y los hábitos de estudio, en alumnos de educación secundaria. REICE, 6(3), 60-99. http://www.rinace.net/arts/vol6num3/art3.pdf

Castro, M. y Morales, M. (2015). Los ambientes de aula que promueven el aprendizaje, desde la perspectiva de los niños y niñas escolares. Revista electrónica Educare, 19 (3), 1-32. http://dx.doi.org/10.15359/ree.19-3.11

Chang, CY y Cheng, WY. (2008). El rendimiento científico y la confianza en sí mismos y la interacción de los estudiantes. Un estudio de muestra representativo de Taiwán. Revista internacional de ciencia Education, (30), 1183-1200.

Cleaves, A. (2005). The formation of science choices in secondary school. International Journal of Science Education, 27(4), 471-486. https://www.tandfonline.com/doi/abs/10.1080/0950069042000323746

Corbin, J. y Strauss, A. (1990). Grounded theory research: Procedures, canons and evaluative criteria. Qualitative Sociology, (13), 3-21.

Cury, F., Biddle, S., Famose, J. P., Goudas, M., Sarrazin, P., y Durand, M. (1996). Personal and situational factors influencing intrinsic interest of adolescent girls in school physical education: A structural equation modelling analysis. Educational Psychology, (16), 305315.

De Vellis, R. F. (2012). Scale Development, Theory and Applications. SAGE.

Derek, Ch. (2018) Los factores clave que afectan el interés individual de los estudiantes en las lecciones de ciencias escolares. International Journal of Science Education, 40(1) 123. https://doi.org/10.1080/09500693.2017.1362711

Dien, J. (2010). Evaluating two-step PCA of ERP data with Geomin, Infomax, Oblimin, Promax, and Varimax rotations. Psychophysiology, 47(1), 170-183.

Etchepareborda, M. (2005). Memoria de trabajo en los procesos básicos del aprendizaje. Revista de Neurología. 40(1), 79-83. http://www.mdp.edu.ar/psicologia/psico/secacademica/asignaturas/aprendizaje/Memoria\%20de\%20trabajo.pdf 
Flick, U. (2007). Introducción a la investigación cualitativa. Sage.

Furman, M. (2018). La educación científica en las aulas de América Latina. ResearchGate, (1), 47-

72. https://www.researchgate.net/publication/330183645

Furr, M. y Bacharach V. (2008). Psychometrics and Introduction. SAGE Publications.

Furr, M. (2011). Scale construction and Psychometrics for social and personality Psychology. SAGE Publications.

Gorsuch, R. L. (1973). Using Bartlett's Significance Test to Determine the Number of Factors to Extract. Educational and Psychological Measurement, (33), 361-364.

Harman, H. H. (1962) Análisis factorial moderno. Prensa de la Universidad de Chicago.

Hasni, A. (2015). Student's Interest in Science and Technology and its Relationships with Teaching Methods, Family Context and Self-Efficacy. International Journal of Environmental \& Science Education, (10), 337-366.

Hendrickson, A. E. y White, P. O. (1964). Promax: a quick method for rotation to oblique simple structure. British Journal of Statistical Psychology, (17), 65-70.

Hernández, A., Muñiz, J. y García, E. (2000). Comportamiento del modelo de respuesta graduada en función del número de categorías de la escala. Psicothema, 12(2), 288-291.

Hidi, S. y Renninger, K. (2006). El modelo de cuatro fases de desarrollo de intereses. Educ Psychol, 41(2), 111-127.

Hogarty, K., Hines, C., Kromrey, J., Ferron, J. y Mumford, K. (2005). The quality of factor solutions in exploratory factor analysis: The influence of sample size, communality, and overdetermination. Educational and Psychological Measurement, (65), 202-226.

Insauti, J. y Merino, M, (2000). Una propuesta para el aprendizaje de contenidos procedimentales en el laboratorio de física y química. Revista lenci, 5(2), 93-119. https://www.if.ufrgs.br/cref/ojs/index.php/ienci/article/view/613/402

Ito, T., y McPherson, E. (2018). Factores que influyen en la escuela secundaria, interes de los estudiantes en PSTEM. Frontiers in Psychology, 9(15). 1-13. https://doi.org/10.3389/fpsyg.2018.01535

Jenkins, E.W. y Pell, R.G. (2006). The relevance of science education proyect (RSE) in england: a summary of findings. Leeds: centre for studies in science and mathematics education. University of leeds.

Kaiser, H. F. (1970). A second generation Little Jiffy. Psychometrika, (35), 401-415.

Kaplan, D. (2009). Structural equation modeling: foundations and extensions. Sage.

Krapp, A. y Prenzel M. (2011). Investigación sobre el interés en la ciencia: teorías, métodos y hallazgos. Revista Internacional de Educación en Ciencias, (33), 27-50.

Krapp, A., Hidi S. y Renninger K.A. (1992). Interest, learning and development. The role of interest in learning and development. Hillsdale, N.J. Erlbaum.

Lorenzo-Seva, U. y Ten Berge, J. M. F. (2006). El coeficiente de congruencia de Tucker como un índice significativo de similitud factorial. Metodología, (2), 57-64.

Marbà, A. y Márquez, C. (2010). ¿Qué opinan los estudiantes de las clases de ciencias? un estudio transversal de sexto de primaria a cuarto de ESO. Enseñanza de las ciencias, 28(1), 19-30. https://ddd.uab.cat/pub/edlc/02124521v28n1/02124521v28n1p19.pdf 
Marsh, HW, Trautwein, U., Lüdtke, O., Köller, O. y Baumert, J. (2005) . Autoconcepto académico, interés, calificaciones y puntajes de pruebas estandarizadas: modelos de efectos recíprocos de ordenamiento causal. Niño Development, (76), 397-416.

Martínez, R. (1995). Psicometría. Síntesis Psicológica.

Moreira, T. (2009). Factores endógenos y exógenos asociados al rendimiento en matemática: un análisis multinivel. Revista de educación, 33(2), 61-80. http://www.redalyc.org/pdf/440/44012058005.pdf

Mohd, E., Halim, L., Sattar, M., Osman, H. y Mohamad, N. (2019) Interés de los estudiantes hacia STEM: un estudio longitudinal. Investigación en ciencia y educación tecnológica, 37(1), 7189. DOI: $10.1080 / 02635143.2018 .1489789$

Mulaik, S. A. (2009). Linear causal modeling with structural equations. Chapman y Hall / CRC. https://doi.org/10.1201/9781439800393

Muthén B. (2004). Latent variable analysis: Growth mixture modeling and related techniques for longitudinal data. In D. Kaplan (ed.), Handbook of quantitative methodology for the social sciences. Sage Publications.

Muthén, L. K. y Muthén, B. O. (1998-2010). Mplus User's Guide. Muthén \& Muthén

Papalia, D., Wendkos, O. y Duskin, f. (2006). Psicología del desarrollo de la infancia a la adolescencia. McGrawHill.

Pérez, J. y Gardey A. (2009). Definición de factor. Definición D. http://definicion.de/factor/

Polino, C. (2012). Las ciencias en el aula y el interés por las carreras científico-tecnológicas: Un análisis de las expectativas de los alumnos de nivel secundario en Iberoamérica. Revista Iberoamericana de educación, 1(58), 167-191. https://doi.org/10.35362/rie580479

Romero, E. y Hernández, M. (2019). Análisis de las causas endógenas y exógenas del abandono escolar temprano: una investigación cualitativa. Educación XXI, 22(1), 263-293. http://revistas.uned.es/index.php/educacionXX1/article/view/21351

Ruiz, J., López, I. y Moreno, C. (2012). Influence of Cooperative Learning on Students' SelfPerception on Leadership Skills: A Case Study in Science Education. Revista higher education studies, 2(4), 40-48. https://files.eric.ed.gov/fulltext/EJ1081293.pdf

San Martin, D. (2014). Teoría fundamentada y Atlas.ti: recursos metodológicos para la investigación educativa. Revista Electrónica de Investigación Educativa, 16(1), 104-122. https://redie.uabc.mx/redie/article/view/727/891

Schiefele, U. (1991). Interest, learning and motivation. Educational Psychologist, 26(3-4), 299232.

Schiefele, U. (1992). Topic interest and levels of text comprehension. Educational Psychologist, 8(2), 141-160. https://www.sciencedirect.com/science/article/pii/S1041608096900308

Schreiner, C. y Sjøberg, S. (2004). Sembrando las semillas de ROSE. Antecedentes, Justificación, Desarrollo de cuestionarios y recopilación de datos para ROSE (The Relevance of Science Educación): un estudio comparativo de las opiniones de los estudiantes sobre la ciencia y la educación científica.

Solbes, J., Montserrat, R. y Furió, C. (2007). El desinterés del alumnado hacia el aprendizaje de la ciencia: implicaciones en su enseñanza. Revista Didáctica de las ciencias experimentales y sociales, 21(1), 91-117. https://file:///C:/Users/DeLL\%20Insp/Downloads/DialnetDesinteresDelAlumnadoHaciaEIAprendizajeDeLaCiencia-2475999.pdf 
Strauss, A. y Corbin, J. (1990) Basics of qualitative research. Sage.

Tabachnick, B. G. y Fidell, L. S. (2007). Using multivariate statistics. Pearson/Allyn \& Bacon.

Tarabini, A., Curran M., Montes A., y Parcerisa L. (2015). La vinculación escolar como antídoto del abandono escolar prematuro: explorando el papel del habitus institucional. Revista de Currículum y Formación de Profesorado, 19(3), 196-212.

Ten Berge, J. M. F. y Kiers, Hal (1991). Un acercamiento numérico al rango mínimo exacto y aproximado de una matriz de covarianza. Psychometrika, (56), 309-315.

Thompson, B. (2008) Exploratory and Confirmatory Factor Analysis, Understanding concepts and applications. American Psychological Association.

Timmerman, M. E. y Lorenzo-Seva, U. (2011). Evaluación de la dimensionalidad de elementos politómicos ordenados con análisis paralelo. Métodos psicológicos, (16), 209-220.

Trumper, R. (2006). Factors Affecting Junior High School Students' Interest in Biology. Science Education International, 17(1). 31-48 http://www.icaseonline.net/seiweb/index.php?option=com content\&view=article\&id= 71:17-1-2006\&catid=39:archive-2005-2007\&ltemid=81

Vázquez, A. y Manassero, M. (2005). La ciencia escolar vista por los estudiantes. Bordon 57(5), 125-143. https://recyt.fecyt.es/index.php/BORDON/article/view/40802

Velicer, W. F. y Fava, J. L. (1998). Effects of variable and subject sampling on factor pattern recovery. Psychological Methods, 3(2), 231-251. https://doi.org/10.1037/1082989X.3.2.231

Whitelegg, E. y Parry, M. (1999). Real-life contexts for learning physics: meanings, issues and practice. Physics Education, 34(2), 68-72.

Viljaranta, J., Tolvanen, A., Aunola, K. y Nurmi, J. (2014). La dinámica del desarrollo entre interés, autoconcepto de capacidad y rendimiento académico. Diario escandinavo de Educational Research, (58), 734-756.

Yanai, H. y Ichikawa, M. (2007). Factor Analysis. En C. R. Rao \& S. Sinharay (editors). Handbook of Statistics 26. Psychometric. Elsevier. 